Review

\title{
The Conserved Molecular Determinants of Virulence in Dengue Virus
}

\author{
Zuleeza Ahmad and Chit Laa Poh ${ }^{\bowtie}$ \\ Centre for Virus and Vaccine Research, School of Science and Technology, Sunway University, 47500 Subang Jaya, Selangor, Malaysia \\ $\triangle$ Corresponding author: pohcl@sunway.edu.my; Tel.: +60-3-7491-8622 (ext. 7338); Fax: +60-3-5635-8633 \\ (C) Ivyspring International Publisher. This is an open access article distributed under the terms of the Creative Commons Attribution (CC BY-NC) license \\ (https://creativecommons.org/licenses/by-nc/4.0/). See http://ivyspring.com/terms for full terms and conditions.
}

Received: 2018.09.14; Accepted: 2018.12.17; Published: 2019.01.24

\begin{abstract}
Dengue virus belongs to the Flaviviridae family which also includes viruses such as the Zika, West Nile and yellow fever virus. Dengue virus generally causes mild disease, however, more severe forms of the dengue virus infection, dengue haemorrhagic fever (DHF) and dengue haemorrhagic fever with shock syndrome (DSS) can also occur, resulting in multiple organ failure and even death, especially in children. The only dengue vaccine available in the market, CYD-TDV offers limited coverage for vaccinees from 9-45 years of age and is only recommended for individuals with prior dengue exposure. A number of mutations that were shown to attenuate virulence of dengue virus in vitro and/or in vivo have been identified in the literature. The mutations which fall within the conserved regions of all four dengue serotypes are discussed. This review hopes to provide information leading to the construction of a live attenuated dengue vaccine that is suitable for all ages, irrespective of the infecting dengue serotype and prior dengue exposure.
\end{abstract}

Key words: dengue virus, virulence, vaccine, mutations, live attenuated vaccine

\section{Introduction}

Dengue virus (DENV) causes approximately 390 million infections per year, of which 100 million are symptomatic infections and 25,000 deaths occur annually, mainly in children [1]. Classified in the same mosquito-borne Flaviviridae family along with the emerging pathogenic viruses such as West Nile virus and Zika virus, DENV also serves as a model organism to facilitate the study of these relatively less-characterised viruses. DENV is endemic in more than 125 countries spanning across South-East Asia, Western Pacific, East Mediterranean, Africa, and the Americas [2]. In South-East Asian countries such as Malaysia, Indonesia and the Philippines, it is of utmost importance to lessen the DENV disease burden as DENV infections have reached epidemic proportion. The DENV mosquito vectors, Aedes aegypti and Aedes albopictus, are also spreading from the tropical and sub-tropical regions to more temperate areas due to global warming [3]. Therefore, it is now more relevant than ever to find an effective prevention strategy against DENV infections and one such strategy is by developing a new live attenuated vaccine which will overcome the limitations encountered by the current licensed DENV vaccine in the market and those under development.

\section{Current DENV vaccine in the market}

The first dengue vaccine, CYD-TDV or Dengvaxia ${ }^{\circledR}$, developed by Sanofi-Pasteur, is a tetravalent, recombinant live attenuated vaccine (LAV) with a yellow fever 17D virus backbone. The four chimeric yellow fever 17D vaccine viruses were engineered to express the surface $\mathrm{E}$ (envelope) and prM (membrane) proteins of each of the four serotypes of dengue virus [4]. In the third year of phase 3 CYD-TDV trial in Asia (the CYD14 trial), there was an excess of hospitalisations for dengue among vaccinated children aged 2 to 5 years [5]. This raised the concern about the potential effects of baseline dengue serostatus and age of the vaccinees on the vaccine safety and efficacy. Nevertheless, CYD-TDV was granted market authorisation starting in 
December 2015 and as of late 2017, was licensed in 19 countries [6].

CYD-TDV is administered in a 3-dose schedule at 6-month intervals and the vaccine has been licensed for individuals aged 9 to 45 years living in dengue endemic areas, although both upper and lower age limits might vary by license. Currently, CYD-TDV is mainly available in the private market except in Brazil and the Philippines, whereby the vaccine was included in their national immunisation programs [7]. There are multiple issues and controversies surrounding the administration of this vaccine. The Philippines government has discontinued its CYD-TDV vaccination program after a number of deaths in children is allegedly linked to CYD-TDV being administered to these children who had no prior dengue infection. Indeed, after 61 months of follow up, Sanofi released new results based on a specifically developed NS1 assay and found that the administration of CYD-TDV increased the risk of hospitalization for dengue in seronegative vaccine recipients compared to controls [8]. By April 2018, WHO issued a report which recommended that the vaccine be administered only to persons who have had a prior dengue infection [6].

The requirement for three doses of CYD-TDV indicated that the immune response to the primary dose of the vaccine was weak. This is most likely due to the fact that CYD-TDV is composed largely of genome sequence derived from the yellow fever virus, and only contained some DENV surface proteins. CYD-TDV also lacked DENV NS1, an immunogenic viral protein secreted at high levels in infected individuals that has been hypothesised to contribute directly to disease pathogenesis [9]. An immune response to NS1 might be essential to protect against DENV. With the apparent lack of T cell epitopes from the non-structural proteins in the CYD-TDV, it is predicted that only neutralising antibodies would be generated in the vaccines while the essential protective $\mathrm{T}$ cell response is missing [10]. All these factors have been stipulated to contribute to the CYD-TDV-enhance disease outcome in seronegative vaccines [11].

\section{Current DENV vaccines in Phase 3 trials}

Two other tetravalent LAVs, Takeda's TDV and National Institutes of Health (NIH)/Butantan Institute's TV003, are undergoing phase 3 trials [12]. TDV consists of attenuated virus with three point mutations (each within the 5'UTR, NS1 and NS3, respectively) in its genome [13]. In Phase 1 studies, following immunisation with TDV, $62 \%$ participants seroconverted in response to all four serotypes while $96 \%$ participants seroconverted in response to at least three dengue virus serotypes [14]. In Phase 2 studies, the two-dose, three-months apart regimen is supported. In participants who were seronegative at baseline, a second dose administered three months later resulted in $86 \%$ seroconversion in response to all four serotypes compared to $69 \%$ in a group which had received only one dose of the vaccine [15]. Takeda has now completed its target vaccinations of 20,000 children and adolescents aged 4 through 16 and preliminary outcomes for these Phase 3 studies are expected by the end of 2019 [16].

The TV003 is comprised of DENV-1, DENV-3, DENV-4 and a chimeric DENV-2 which all carried a 30-nucleotide deletion in the $3^{\prime}$ UTR of its genome [17]. Previously, the vaccine provided robust and balanced immune responses to all four serotypes by inducing a tetravalent antibody response in $74 \%$ of the healthy, flavivirus-naïve participants, with 92, 76, 97, and $100 \%$ of the vaccinees seroconverting in response to DENV-1, DENV-2, DENV-3, and DENV-4, respectively [18]. In the follow up study, the ability of a single dose of TV003 to protect against DENV challenge was evaluated. After a single dose of TV003, followed by challenge with the DENV-2 virus rDEN2D30 six-month post-vaccination, all 21 recipients were protected from infection by rDEN2D30. In contrast, $100 \%$ of the 20 placebo recipients who were challenged with rDEN2D30 developed viraemia, 80\% developed rash, and 20\% developed neutropenia. [19]. Additionally, TV003 induced $87 \%$ seroconversion in response to all four serotypes of DENV in participants with prior flavivirus exposure. However, it is unknown whether the induced neutralising antibodies recorded were sufficient to confer protection against natural dengue infection. The mean peak antibody titres of the recruited individuals also gradually declined six-month post-vaccination although not to as low as the baseline levels [20]. An estimated 16,944 subjects aged 2 to 59 years old are currently being recruited for the Phase 3 trial to evaluate the efficacy and safety of TV003 (ClinicalTrials.gov Identifier: NCT02406729). It was also noted that due to the DENV-2 component being less immunogenic than the other serotypes in TV003, TV005 was formulated to have exactly the same formulation as TV003 except that it contained ten times more of the DENV-2 component [18]. TV005 has yet to progress to Phase 3 trials although it has shown promising results in terms of its magnitude of immune response, HLA restriction and antigen specificity [21]. The TV003/TV005 vaccine is planned to be administered as a single subcutaneous dose.

For TDV, the genetic stability of the three point mutations ( 5 ' UTR-57, C to T; NS1-53 glycine to aspartate; NS3-250 glutamate to valine) is of concern. 
The 5`UTR-57-T showed high propensity of reversion to the pathogenic wild type at very low passage numbers (as few as ten passages) [22]. Due to the limited number of mutations in TDV, there is a strong possibility that the attenuated vaccine strain could revert to its wild type form [23]. The genetic stability of the 30-nucleotide deletion in TV003/TV005 vaccine is not yet evaluated, however the vaccine has encountered a potential setback as lower seroconversion rate in African Americans (57\%) was recorded following administration of the vaccine when compared to non-African Americans (86\%) [24].

Construction of the TDV vaccine strain through attenuation has been achieved through serial passages of the wild type virus in cell lines, in particular using primary dog kidney (PDK) cells [25]. These attenuated strains have been tested separately for safety and immunogenicity before being formulated into a tetravalent product. Unfortunately, it appeared that once formulated into tetravalent vaccines, some level of interference between the virus strains occurred, leading to a dampened or adverse immune response for some DENV serotypes as compared to the monovalent vaccine candidates [26]. For example, DENV-3 vaccine strain DENV-3 PGMK30 was generated by passaging the wild type DENV-3 16562 thirty times in primary green monkey kidney cells and three times in primary fetal rhesus lung cells. In a human safety trial, however, all volunteers who received the tetravalent formulation containing PGMK30 developed symptoms and signs consistent with acute dengue, with detectable DENV-3 viraemia [27]. Following the disappointing clinical trial results, this DENV-3 vaccine strain was further passaged in Vero cells using the same protocol to yield the Vero-derived Vaccine (VDV3) strain, which showed attenuation as the PGMK30 strain previously. When VDV3 was administered to healthy volunteers, it again caused disease in recipients [28,29].

Hence, understanding the exact molecular determinants of virulence that contributed to these divergent clinical outcomes despite the observed attenuation in vitro could enable a more objective and accurate approach in selecting attenuated strains for development into LAVs. Notably, CYD-TDV as the only licensed vaccine against DENV is not approved for children younger than 9 years and children are the main age group which is the most vulnerable to DENV infection. The need remains for a vaccine that is safe and effective in recipients of all ages, especially those younger than 9 years. In summary, an ideal DENV vaccine should be safe for both seronegative and seropositive individuals, attenuated in such a way that is stable with no possibilities of reversion to its wild type phenotype and be sufficiently immunogenic to confer long-life protection and broad protection against all four DENV serotypes. Therefore, the identification of molecular determinants of DENV virulence within the conserved regions of the genomes of all four DENV serotypes will aid in vaccine design. Such considerations when constructing the next LAV against dengue is more likely to confer a balanced and protective immunity against DENV, independent of its serotypes and be capable of inducing high seroconversion in vaccinees, irrespective of prior dengue exposure [30,31].

\section{The molecular determinants of virulence in dengue virus}

The four antigenically-distinct dengue virus serotypes, DENV-1 to 4, share 60 to $70 \%$ genetic homology [32]. Initial infections with any of the four DENV serotypes only raise protective serotypespecific antibodies. The dominant population of antibodies against one DENV serotype, while crossreactive, are non-neutralising towards other DENV serotypes [33]. This contributes to the manifestation of a more severe disease if one is to be re-infected by a heterologous DENV serotype, a phenomenon known as antibody dependent enhancement (ADE) of dengue infection. Indeed, $\mathrm{ADE}$ is thought to be the underlying reason as to why there is a high rate of infants showing severe dengue disease during their first dengue infection as the levels of maternal polyclonal dengue antibodies would start to wane after birth $[34,35]$.

\section{DENV morphology and genome organisation}

All flaviviruses including DENV share a similar organisation of the virion and genomic structure [36]. Each virion is spherical in shape, enveloped and about $50 \mathrm{~nm}$ in size. DENV envelope is comprised of the envelope (E) protein which is organised into dimers and the membrane $(\mathrm{M})$ protein which is cleaved from the immature prM protein by the host protease, furin. Inside the envelope is the nucleocapsid, which consists of multiple copies of the capsid (C) protein and the viral genome. The viral genome is a 10.7 kilobase, positive-sense, single- stranded, capped RNA (+ssRNA). The viral proteins are encoded in a single open reading frame (ORF) that is co- and post-translationally cleaved by viral and host proteases and is flanked by $5^{\prime}$ and $3^{\prime}$ untranslated regions (UTRs). Overall, the ORF encodes for three structural proteins (C, prM, E), and seven non-structural (NS) proteins [37].

Genetic comparisons of strains with varying degrees of pathogenicity have revealed differences in 
the nucleotide and polyprotein sequences, suggesting that they play a role in virulence. Here we present the specific molecular determinants within the DENV genome and the amino acids of the DENV polyprotein that appear to have a significant role in virulence, either in vitro and in vivo. These are only discussed here if they are highly conserved in all four serotypes and are not included in the Sanofi Pasteur CYD-TDV, Takeda TDV and NIH TV003/TV005 vaccines. Any mutations that are known and tested to be unstable, led to replicative-defective phenotype and secondary mutations to arise are not included in this review as these mutations are not going to be suitable for a LAV development.

\section{5' untranslated region (5' UTR)}

The non-coding $5^{\prime}$ untranslated region (5' UTR) spans approximately 100 nucleotides and shows almost complete sequence conservation among the four serotypes [38]. The identified elements within the $5^{\prime}$ UTR include the large 5' stem loop A (5' SLA) and the short $5^{\prime}$ stem loop B (5' SLB) which ends in the translation initiation AUG codon [39, 40]. These elements have been shown to be required for virus replication, translation and pathogenesis. For instance, SLA was shown to be necessary for the correct positioning of the NS5 RNA polymerase before the initiation of RNA synthesis [41]. On the other hand, SLB contains a sequence known as 5' UAR (Upstream AUG Region) that is complementary to a sequence located at the 3' UTR of the viral genome [42].

Sirigulpanit et al. [43] demonstrated that DENV with a single substitution mutation at nucleotide position 69 from $A$ to $U$, contributed to a lower mortality rate in mice, but yielded similar plaque size compared to the wild type. The nucleotide at position 69 is located within the region in between SLA and SLB of the 5' UTR. Previously, Leitmeyer et al. [44] discovered that the same exact substitution mutation from A to $U$ at the nucleotide position 69 in the $5^{\prime}$ UTR was among the changes that distinguished the more virulent South-East Asian genotype from the American genotype. Despite the fact that the sequences obtained from the first 68 nucleotides were identical among the two strains, those belonging to the American genotype had a different nucleotide at positon 69. This is predicted to change the secondary structure, resulting in a reduced stem length, and a longer 39-terminal loop of 12 nucleotides compared to the more virulent Southeast Asian genotype. Next, Cahour et al. [40] showed that deletion of nucleotides from position 82-87 which resides within the SLB in the 5' UTR yielded viable viruses with reduced translation efficiency. The growth-restricted mutant progeny produced small plaques in simian LLC-MK2 cells but failed to produce any plaque in mosquito C6/36 cells.

\section{Capsid (C)}

The $\mathrm{C}$ protein contains a flaviviral-conserved internal hydrophobic domain which may be associated with the host ER membrane. This interaction is thought to facilitate the assembly and the entry of the virus into the lumen of host ER as virions. The removal of the internal hydrophobic region of protein $C$ at amino acid positions 42-59 led to a significant attenuation in the suckling mice and this is likely due to the disruption in the assembly of infectious viral particles [45].

Within the capsid-coding region lies a stem-loop structure known as the capsid-coding region hairpin element (cHP). cHP enhances translation start codon selection and is required for viral replication [46]. Clyde et al. [47] showed that any mutations that disrupt $\mathrm{CHP}$ are rescued by spontaneous compensatory mutations that restabilised the cHP structure. One such mutation is at nucleotide position 204, located well downstream of the cHP. At this position, $A$ is substituted into $G$ with no change in the resulting amino acid sequence. Although the cHP structure was rescued, the A204G infectious clone retained a defect in viral replication [47].

\section{Pre-membrane/membrane (prM/M)}

Pryor et al. [48] introduced a number of mutations at amino acid residue position 39 in the prM coding region. Comparison of $M$ protein sequences of 40 flaviviruses revealed that a polar amino acid is always present at position 39. For DENV-1, -2 and -3 , the residue at M-39 is histidine, whereas for DENV-4 and all other flaviviruses, it is asparagine. They found that a substitution of the histidine residue with the basic amino acid arginine in the DENV-2 genome resulted in a moderate but significant $(\sim 2 \log 10)$ reduction in virus titre. A substitution of histidine-39 with uncharged polar amino acid glutamine was also shown to severely decrease viral replication [48]. Indeed, a histidine to arginine substitution at residue 39, in addition to two changes in the $\mathrm{E}$ protein, was identified in a chimeric DENV-1/yellow fever virus and it caused decreased viraemia in monkeys compared to the parental DENV-1 and yellow fever virus [49].

After proteins required for virus assembly are translated, the prM forms heterodimers with E protein intracellularly before immature virus particles can be secreted [50]. The C-terminus of prM protein is consisted of an a-helical domain (MH) (residues 113 to 128), followed by two transmembrane domains 
(MT1 and MT2) [51]. The role of nine highly conserved $\mathrm{MH}$ residues in the replication cycle of DENV in a DENV-1 prME expression construct was investigated by Hsieh et al. [52]. Alanine substitutions on all nine residues studied except for alanine substitution on residue 112 were found to impair prM to $\mathrm{M}$ cleavage by furin and therefore, would lead to the production of non-infectious virus-like particles (VLPs) [53]. VLPs have been proposed to be promising vaccine candidates against flaviviruses such as Murray Valley encephalitis virus [54] and also DENV [55]. However, for the purpose of LAVs development, mutations that led to the production of VLPs are not suitable as an attenuation method as VLPs will not be able to replicate in the host. Interestingly, one out of the nine mutants constructed by Hsieh et al. [52] which had a substitution of serine to alanine at position 112 within the M polypeptide demonstrated impaired viral assembly. The mutation did not affect the prM cleavage and replicon particle entry, making it an ideal mutation to be incorporated into a DENV LAV strain [52].

\section{Envelope (E)}

The E glycoprotein, situated on the surface of the dengue virion, is responsible for virus attachment, virus-specific membrane fusion in acidic $\mathrm{pH}$ endosomes, and subsequent virus assembly. Butrapet et al. [56] engineered fifteen mutant dengue viruses to identify amino acids in one of the molecular hinges of the envelope protein that are critical for viral infection. One such amino acid is an alanine residue at position 54 which is also conserved in all flaviviruses. Mutation of alanine to valine at residue 54 from the wild type yellow fever French viscerotropic virus in the French neurotropic vaccine (FNV) strain was postulated to be one of the $\mathrm{E}$ protein mutations contributing to the attenuation phenotype of FNV [57, 58]. Therefore, Butrapet et al. [56] substituted the small hydrophobic residue alanine with the large acidic residue glutamic acid. This mutation resulted in a shifted fusion threshold to a higher $\mathrm{pH}$ and caused slightly delayed replication in Vero cells, suggesting alanine-54 might be important for stabilisation of the functional hinge structure. Additionally, threonine at position 280 was also targeted for a substitution with a much larger tyrosine. This led to altered fusion threshold to a slightly lower $\mathrm{pH}$ and the mutant showed significantly lower fusion capacity than the wild type virus [56].

Huang et al. [59] conducted a comprehensive study of the E protein which is known to direct virus-mediated membrane fusion. They introduced 27 mutations into the fusion peptide of an infectious cDNA clone of DENV-2 and recovered seven stable mutant viruses. The fusion efficiency of the mutants was impaired, demonstrating the requirement for specific fusion peptide amino acids for optimal fusion. The mutant viruses (G102S, G104S, F108W) exhibited different growth kinetics and/or genetic stabilities in different cell types and adult mosquitoes. Virus particles could be recovered following RNA transfection of cells with five mutants (W101F, L107A, F108A and WF101, 108FW); however, recovered viruses could not re-infect cells. These viruses could enter cells, but internalised virus appeared to be trapped in endosomal compartments of infected cells, thus suggesting a fusion blockade.

The ability of DENV to escape the endosome and release its nucleocapsid into the cytoplasm for replication prior to lysosomal degradation is critical during virus entry via clathrin-mediated endocytosis in non-ADE infection. However, during ADE of DENV infection however, virus-antibody complexes binding to $F_{C \gamma} R$ is thought to allow the virus antibody complexes to enter cells through phagocytosis. As virions that enter by $\mathrm{F}_{\mathrm{c}} \mathrm{R}$-mediated phagocytosis would still eventually enter the endosomal/lysosomal pathway, viral-endosomal membrane fusion is likely necessary for the infectivity of virus-antibody complexes [60]. Therefore, Chotiwan et al. [61] used several DENV-2 E protein mutants to identify molecular determinants critical for virus-immune complex entry via $F_{c}$ RIIA-bearing human myelogenous K562 cells and monkey CV-1 fibroblasts constitutively expressing transfected human FcyRIIA. Indeed, using two temperature-sensitive, fusion-defective mutant viruses carrying mutations (G104S and L135G) targeted to affect different steps in fusion, they demonstrated that the DENV E protein conformationmediated membrane fusion is critical for infectivity under both ADE and non-ADE conditions.

The flexible molecular hinges of the envelope protein as mentioned earlier is important for virion assembly and entry, specifically as it enables the correct conformational changes of the $\mathrm{E}$ protein to occur for fusion and maturation process [62, 63]. Initially, Goo et al. [64] found that a T198F mutation in the envelope protein of West Nile virus resulted in a $\sim 70$-fold increase in sensitivity to neutralization by a monoclonal antibody, E60. This monoclonal antibody is known to target a cryptic epitope that is normally poorly accessible [65]. Therefore, it follows that the epitope was exposed more in the T198F mutant, leading to reduced virus stability at physiological temperatures. Based on sequence homology, the threonine at residue 198 in West Nile virus corresponds to a phenylalanine residue in DENV at position 193. The introduction of the reciprocal mutation F193T in the Western Pacific strain of 
DENV-1 led to $\sim 10$-fold reduction in the infectivity and $\sim 3$-fold reduction in the half-life of the virus particles. Similar to results with WNV T198F, the mutant DENV-1 F193T was more sensitive to neutralization by the monoclonal antibody E60 than the wild type viruses. Hence, it appears that a single point mutation at a molecular hinge of the $\mathrm{E}$ protein of two flaviviruses, DENV and West Nile virus, can alter the $\mathrm{E}$ protein conformation such that the mutant viruses became less virulent and stable. More importantly, the T198F change in the West Nile virus resulted in the attenuated phenotype in mice, therefore, it will be imperative to investigate whether this also applies to DENV [64].

\section{NS proteins}

The DENV NS proteins, except for NS5, are integral membrane proteins. Flavivirus NS1 is a 48 $\mathrm{kDa}$ glycoprotein which is composed of five transmembrane domains that is translocated into the ER lumen co-translationally [66]. NS1 plays an array of functions. Aside from facilitating virus replication, it can also be secreted and is thought to help the virus evade the immune system [67, 68]. NS1 also modulated early events in viral RNA replication and was shown to co-localise with double stranded RNA (dsRNA) and interacted with the NS4B [69]. More recently, it was demonstrated that NS1 interacted with the E glycoproteins presumably on the surface of virions and these interactions were required for efficient production of infectious virus particles [70].

In the infected cell, NS1 is bound to the membrane of the ER vesicles on the lumen side and dimerises upon the addition of high-mannose carbohydrates to help anchoring the viral replication complex. The NS1 dimer contains three domains; a small $\beta$-roll domain, a Wing domain, and a $\beta$-ladder domain [71]. Scaturro et al. [70] selected 46 residues for alanine scanning mutagenesis and found that alanine substitutions at position S114, W115, D180 or T301 of NS1 altered the production of infectious virus. Specifically, the mutants showed minor effects of viral RNA replication, but the virus production was greatly impaired. The mutants also had reduced intracellular and extracellular infectivity. The study supported the notion that NS1 is a critical determinant for the assembly or release of infectious virus particles.

Both NS2A and NS2B are very poorly characterised so far. Mis-cleavage between NS1 and NS2A could affect viral RNA replication, and mutations in the protein was observed to affect virion assembly. A mutagenesis study revealed that several NS2A mutations, G11A, E20A, E100A, Q187A, and K188A, impaired virion assembly without specifically affecting viral RNA synthesis [72]. In addition, the
NS2A protein of DENV has eight predicted transmembrane segments. By introducing a series of triple alanine substitutions, Wu et al. (2017) created six NS2A mutants with mutations within the transmembrane segment, one to two of which displayed novel phenotype. These mutants showed a $>1,000$-fold reduction in virus yield and were unable to form plaques despite having wild type-levels of viral replication and no defects in virus assembly and secretion. One of the mutants, NM-5, had alanines replacing the semi-conserved alanine-lysine-phenylalanine residues. Overall, the findings suggest that the amino acid sequence of the N-terminal half of DENV2 NS2A is critical for viral RNA synthesis and cytopathic effects [73]. NS2B is a cofactor for NS3 protease and it co-localises with dsRNA, NSI, NS3, and NS5 within the replication complex. A 40-amino-acid segment of NS2B (DENV-4 amino acids 1396 to 1435) comprising a hydrophilic domain surrounded by hydrophobic regions was found to be essential for NS2B-NS3 serine protease activity [74].

NS3 has two domains: the protease lies at the $\mathrm{N}$-terminal end and requires the NS2B cytoplasmic loop [75, 76] while the C-terminal domain of NS3 possesses the helicase activity that presumably unwinds double-stranded RNA along with an ATPase activity involved in the capping of the newly synthetized genomic RNA [77, 78]. Blaney et al. [79] analysed the recombinant DENV-4 viruses which were temperature sensitive and found that they yielded small plaques and were attenuated in the suckling mice. One of the mutants was a NS3 mutant with a substitution at position 192 from aspartic acid to asparagine. Additionally, this mutant was also found to confer a greater than 10,000-fold reduction in replication of the recombinant virus in SCID mice transplanted with HuH-7 cells [79].

NS4A contains two transmembrane domains and the first 48 amino acids of DENV-2 NS4A were reported to form an amphipathic helix that mediates oligomerisation [80, 81]. NS4A is thought to act as a scaffold for the replication complex and is proposed to induce membrane alterations. Following nuclear magnetic resonance analysis, Lee et al. [82] constructed alanine substitutions for 15 flaviviruses with conserved NS4A residues and found that two amino acids located within the first transmembrane domain are important for viral replication, NS4A oligomerisation and stability. On the other hand, NS4A also interacted with NS4B in virus-infected cells and in three other NS4A mutants, L48A, T54A, and L60A with disrupted NS4A-NS4B interactions, abolished or severely reduced viral replications were demonstrated [83]. 
Table 1. Nucleotide and amino acid changes in the genome of DENV that contributes to virulence.

\begin{tabular}{|c|c|c|c|c|c|}
\hline & $\begin{array}{l}\text { Region of } \\
\text { genome }\end{array}$ & Position in the genome & Mutation & Effects of the mutation & References \\
\hline 1 & $5^{\prime}$ UTR & Nucleotide 69 in DENV-2 & A to $\mathrm{T}$ & $\begin{array}{l}\text {-Reduced mice mortality rate } 31.25 \% \text { compared to the wild type }(84.37 \%) \\
\text {-Distinguished the South-East Asian genotype from the American genotype }\end{array}$ & {$[43,44]$} \\
\hline 2 & $5^{\prime}$ UTR & Nucleotide $\Delta(82-87)$ in DENV-4 & 6 nt deletion & $\begin{array}{l}\text {-Reduction in translation efficiency } \\
\text {-Yielded small plaques in simian LL2-MK2 cells, no plaques in C6/36 cells }\end{array}$ & [40] \\
\hline 3 & $\mathrm{C}$ & Nucleotide (42-59) in DENV-2 & 19 nt deletion & -Mutant highly attenuated in the suckling mice & [45] \\
\hline 4 & $\mathrm{C}$ & Nucleotide 204 in DENV-2 & A to $G$ & -Reduced viral replication by at least $2.5-4.0 \mathrm{log}$ units & [47] \\
\hline 5 & $\mathrm{pRM}$ & Amino acid 39 in DENV-2 & $\mathrm{H}$ to $\mathrm{R}$ & $\begin{array}{l}\text {-Reduced virus titres (1.7-2.2 log10 lower than the parental virus) Decreased } \\
\text { viraemia in monkeys }\end{array}$ & {$[48,49]$} \\
\hline 6 & $\mathrm{pRM}$ & Amino acid 39 in DENV-2 & $\mathrm{H}$ to $\mathrm{Q}$ & $\begin{array}{l}\text {-Severe effect on virus replication, with overall } 5.3 \log 10\left(33^{\circ} \mathrm{C}\right) \text { or } 4.2 \log 10 \\
\left(37^{\circ} \mathrm{C}\right) \text { decreases in final virus titres }\end{array}$ & [48] \\
\hline 7 & prM & Amino acid 112 in DENV-1 & $\mathrm{S}$ to $\mathrm{A}$ & $\begin{array}{l}\text {-Reduced the assembly of replicon particles } \\
\text {-Showed more than one log reduction in the amounts of replicon RNA }\end{array}$ & [52] \\
\hline 8 & $\mathrm{E}$ & Amino acid 54 in DENV-2 & A to $\mathrm{E}$ & -Lower replication efficiency in Vero cells & [56] \\
\hline 9 & $\mathrm{E}$ & Amino acid 54 and 280 in DEV-2 & A to $\mathrm{E}$ and $\mathrm{T}$ to $\mathrm{Y}$ & -Altered the $\mathrm{pH}$ threshold of fusion & [56] \\
\hline 10 & $\mathrm{E}$ & $\begin{array}{l}\text { Amino acid 102, } 104 \text { and } 108 \text { in } \\
\text { DENV-2 }\end{array}$ & G to $S, G$ to $S, F$ to $W$ & -Reduced growth in mammalian cells at $37^{\circ} \mathrm{C}$ by 2 to more than 4.6 logs & [59] \\
\hline 11 & $\mathrm{E}$ & $\begin{array}{l}\text { Amino acid 101, 107, } 108 \text { in } \\
\text { DENV-2 }\end{array}$ & $\begin{array}{l}W \text { to } F, L \text { to } A, F \text { to } \\
A, W \text { to } F, F \text { to } W\end{array}$ & $\begin{array}{l}\text {-Showed amplification of vRNA and viral antigen expression in V-0 } \\
\text { transfections, but failed to produce virus that could initiate a second round (V-1) } \\
\text { of infection }\end{array}$ & [59] \\
\hline 12 & $\mathrm{E}$ & Amino acid 108 in DENV-2 & $\mathrm{F}$ to $\mathrm{A}$ & $\begin{array}{l}\text {-Mutant was able to enter endosomes through endocytosis but unable to escape } \\
\text { from the endosomal and lysosomal compartments }\end{array}$ & [59] \\
\hline 13 & $\mathrm{E}$ & Amino acid 104 and 135 in DENV-2 & G to $S$ and $L$ to $G$ & -Deficient viral fusion activity during ADE conditions & [61] \\
\hline 14 & $\mathrm{E}$ & Amino acid 193 in DENV-1 & $\mathrm{F}$ to $\mathrm{T}$ & $\begin{array}{l}\text {-Led to } \sim 10 \text {-fold reduction in the infectivity } \\
\text {-Led to } \sim 3 \text {-fold reduction in the half- life of the virus particles. } \\
\text { - The mutant viruses were more sensitive to neutralization by the monoclonal } \\
\text { antibody E60 than the wild type viruses }\end{array}$ & [64] \\
\hline 15 & NS1 & $\begin{array}{l}\text { Amino acid 114, 115, } 180 \text { and } 301 \text { in } \\
\text { DENV-2 }\end{array}$ & $\begin{array}{l}\mathrm{S} \text { to } \mathrm{A}, \mathrm{W} \text { to } \mathrm{A}, \mathrm{D} \text { to } \\
\mathrm{A} \text { and } \mathrm{T} \text { to } \mathrm{A}\end{array}$ & $\begin{array}{l}\text {-Minor effects on RNA replication, but massive impairment of virus production } \\
\text { (up to } \sim 2.5 \log 10 \text { reduction compared to the wild type) }\end{array}$ & [70] \\
\hline 16 & NS2A & $\begin{array}{l}\text { Amino acid 11, 20, 100, 187, and } 188 \\
\text { in DENV-2 }\end{array}$ & $\begin{array}{l}\text { G to } A, E \text { to } A, E \text { to } \\
A, Q \text { to } A \text { and } K \text { to } A\end{array}$ & -Impaired virion assembly without specifically affecting viral RNA synthesis & \\
\hline 17 & NS2A & Amino acid 31-33 & ALF to AAA & $\begin{array}{l}\text { - Showed }>1,000 \text {-fold reduction in virus yield, an absence of plaque formation } \\
\text { despite wild-type-like replicon activity, and infectious- virus-like particle yields. }\end{array}$ & [73] \\
\hline 18 & NS2B & Amino acid 54-92 in DENV-4 & 40 aa deletion & -Eliminated autoproteolytic activity & [74] \\
\hline 19 & NS3 & Amino acid 192 in DENV-4 & $\mathrm{D}$ to $\mathrm{N}$ & $\begin{array}{l}\text { - Attenuated virulence as measured from suckling mice brain and SCID-Huh-7 } \\
\text { serum }\end{array}$ & [79] \\
\hline 20 & NS4A & Amino acid 50 and 67 in DENV-2 & E to $A$ and $G$ to $A$ & $\begin{array}{l}\text {-Reduced viral replication } \\
\text {-Decreased NS4A oligomerisation } \\
\text {-Reduced NS4A protein stability }\end{array}$ & [82] \\
\hline 21 & NS5 & $\begin{array}{l}\text { Amino acid } 325,519,769,840 \text { and } \\
841 \text { in DENV-2 }\end{array}$ & $\begin{array}{l}R \text { to } A, R \text { to } A, R \text { to } \\
A, K \text { to } A \text { and } R \text { to } A\end{array}$ & -High RNA synthesis activity in vitro but delayed or impaired replication in vivo & [91] \\
\hline 22 & NS5 & $\begin{array}{l}\text { Amino acid } 362 \text { and } 370 \text { in } \\
10418-10422 \text { in DEN-2 }\end{array}$ & $\mathrm{R}$ to $\mathrm{A}$ and $\mathrm{K}$ to $\mathrm{A}$ & -Delayed viral replication and reduced polymerase activity in vitro & [91] \\
\hline 23 & 3' UTR & $\begin{array}{l}\text { Nucleotide } 10336-10340 \text { and } \\
10418-10422 \text { in DEN-2 }\end{array}$ & $\begin{array}{l}\text { CTAC to GATA and } \\
\text { CCTG to GGAC }\end{array}$ & $\begin{array}{l}\text {-Produce shorter sfRNAs that showed reduced viral fitness in human cells, } \\
\text { quickly outcompeted by strains that generate the long sfRNAs. }\end{array}$ & [99] \\
\hline 24 & $3^{\prime}$ UTR & $\begin{array}{l}\text { Nucleotide } 10474-10478 \text { and } \\
10562-10566 \text { in DENV-2 }\end{array}$ & 5 nt deletion & -Reduce viral translation rate by $60 \%$ & [95] \\
\hline
\end{tabular}

Note: For 5' UTR and 3' UTR, the number represents the nucleotide positions on the respective DENV genome and for C to NS5, the number denotes amino acid residue position from the $\mathrm{N}$-terminus of the respective protein.

NS4B contains three transmembrane domains and is N-glycosylated at residues 58 and 62 [84, 85]. To the best of our knowledge, no stable and non-lethal mutations in the NS4B protein have been discovered. One mutant which contained a proline to leucine mutation in amino acid 101 of the NS4B did result in decreased replication in $\mathrm{C} 6 / 36$ cells relative to the wild type DENV-4 and decreased infectivity for mosquitoes. However, this mutant showed enhanced replication in Vero and human $\mathrm{HuH}-7$ cells, and enhanced replication in SCID mice implanted with HuH-7 cells which rendered the mutation inappropriate to be included in a vaccine strain [86].

NS5 is the largest and most highly conserved flavivirus protein. The N terminus of NS5 contains an $\mathrm{N}$-terminal methyltransferase domain (MTase) and it has been suggested that this domain is involved in the methylation of the $5^{\prime}$ cap [87]. The capping of the viral RNA genome is required for its stability and translation into viral polyproteins by host cell ribosomes [88]. The NS5 C-terminal contains the RNA-dependent RNA polymerase (RdRp) domain and previous studies have demonstrated that DENV genome cyclisation was necessary for relocating the promoter-NS5 complex with the RdRp formed at the $5^{\prime}$ end, to the $3^{\prime}$ end initiation site [41]. NS5 also appeared to interfere with the innate antiviral cell response by binding and inducing STAT2 degradation [89]. In addition, NS5 interacted with the viral NS3 protein by stimulating the nucleoside triphosphatase (NTPase) and RNA triphosphatase activities of NS3 [90]. 
A mutational analysis of the RdRp domain of DENV-2 NS5, in which basic residues on the surface of the protein were replaced with alanine residues, generated a number of NS5 mutants (R325A, R519A-K523A, R769A, K840A-R841A). These mutants had high RNA synthesis activity in the recombinant protein but delayed or impaired replication when introduced into an infectious clone. This suggests that the residues could interfere with the other functions of NS5 apart from its polymerase activity. Interestingly, another mutant (R361A-K370A) showed slow-replication phenotype which was correlated with its reduced in vitro polymerase activity. Both residues 361 and 370 are thought to reside in the nuclear localisation site, hence mutations on these sites might have caused the NS5 mis-localisation [91]. Binding of the stem loop A (SLA) at 5' UTR to the RdRp domain of NS5 was not sufficient to drive the polymerase activity; this process required specific contacts between the SLA and the RdRp [92].

\section{$3^{\prime}$ untranslated region ( $3^{\prime}$ UTR)}

The DENV 3' untranslated region (3 'UTR) is approximately 400 nucleotides in length and lacks a poly(A) tract [93]. It comprises two stem loops xrRNA1 and xrRNA2, strengthened by pseudoknots (PK 1 and 2) in Domain I (variable region). Domains II and III consist of two dumbbell (DB) structures and a stem loop (SL) which are more conserved [94]. The two dumbbell structures DB1 and DB2 have the tendency to form two potential pseudoknots between identical five-nucleotide terminal loops 1 and 2 (TL1 and TL2) and their complementary pseudoknot motifs, PK2 and PK1. Manzano et al. [95] created a deletion of five nucleotides within the TLl and TL2 in the 5' DB and 3' DB, respectively. Consequently, the viral translation rate was reduced by $60 \%$ in the TLl/TL2 double deletion mutant. This suggested that TLl acted synergistically with TL2 in viral translation [95]. Proutski et al. [96] showed that the length of sequence deletion is inversely correlated with infectivity. They also suggested that the structure rather than sequence is important for 3' UTR function.

It was recently established that non-coding RNAs tend to accumulate during flavivirus infections. These non-coding RNAs are known as subgenomic flavivirus RNAs (sfRNAs). sfRNAs are the products of incomplete viral genome degradation of the viral 3' UTR by the host exonuclease, $\operatorname{Xrn1}[97,98]$. In a recent study, Filomatori et al. [99] found that by adapting DENV-2 in either mosquito or human cell line, different patterns of sfRNAs would be produced. The shorter variants, sfRNA3 and sfRNA4, were positively selected in the mosquito cell line compared to the wild type-like longer sfRNA1, which was the main sfRNA species detected in human cell line-adapted DENV-2. It was demonstrated that sfRNA3 and sfRNA4 were produced as the result of stalling degradation just upstream of two dumbbell structures, DB1 and DB2, respectively within the DENV-2 3' UTR. Point mutations within a stem-loop structure preceding DB1 and DB2 were found to be sufficient to emulate mosquito-adapted DENV-2 behaviour by generating mainly sfRNA3 and sfRNA4. The mutants which carried the point mutations, S4 and S6, exhibited reduced fitness in human cell lines, induced higher type 1 interferon responses and could be easily outcompeted by viruses that generate the long sfRNA1 [99].

\section{Conclusion}

In conclusion, developing a vaccine for DENV is arguably a challenging task because DENV infection tend to manifest in a more severe form after a previous infection with a heterologous DENV serotype. Additionally, the four serotypes of DENV could also be co-circulating at different prevalence rates in the same region and the pattern of DENV serotypes circulating in a region could also be vastly different from another region [100]. The only available vaccine, CYD-TDV, appears to only elicit antibodies that are unable to neutralize heterologous DENV serotypes. Hence, this has drawn attention to the need to understand the underlying mechanism(s) of protection conferred by both humoral and cellular immunity in the context of a dengue vaccine design.

Before advancing new LAV against DENV to field trials, it might be advantageous to quantitatively analyse the molecular determinants of virulence and the stability of the mutations in the genome both in vitro and in vivo. Each of the monovalent component of the potential vaccine strains must also be tested in seronegative human volunteers [101]. This review has identified a number of conserved molecular determinants of virulence in DENV by identifying the nucleotides and amino acids responsible for reduced DENV virulence in vitro and/or in vivo. Given the conserved nature of all the nucleotides and amino acids discussed here, it follows that the mutations in these nucleotides and amino acids should theoretically be able to reduce DENV virulence in all four DENV serotypes. We speculate that construction of a set of such mutations that are well distributed along the DENV genome would be more likely to produce a successful vaccine candidate. Maintaining the backbone of each DENV serotype and incorporating the same exact set of key mutations into the genome of each monovalent strain in a tetravalent formulation might be the ideal solution to minimise the interference between different viral strains. It is 
likely that the chimeric DENV-2 might have replicated faster in TV003 due to its DENV-4 backbone.

Additionally, maintaining the backbones of all the four DENV serotypes is crucial as we now learned that $\mathrm{CD}^{+} \mathrm{T}$ cells predominantly target conserved non-structural (NS) epitopes of DENV [102, 103] while $\mathrm{CD}^{+} \mathrm{T}$ cells target predominantly the capsid, NS3 and NS5 epitopes [21]. NS genes are also more conserved than structural genes. Hence, it is possible that by following our proposed rational vaccine design of a multiply-mutated tetravalent DENV vaccine, this will induce the production of memory $\mathrm{T}$ cells that target all the serotypes [20]. Since high levels of neutralising antibodies will be elicited, they will be able to neutralise the DENV and prevent enhancement of heterotypic virus infection through $\mathrm{Fc}_{\mathrm{c}} \mathrm{R}$ bearing cells [104]. This should ensure that the vaccine is sufficiently immunogenic regardless of the individual's prior DENV exposure and the infecting serotype which in turn will lower the risk of ADE. Undoubtedly, this step may require a number of trials and errors in order to identify which combinations of the mutations could synergistically lead to more pronounced reductions in virulence. It should also be emphasised that a LAV development against DENV will benefit greatly from prominent advancement in diagnostics tools as current assays do not easily distinguish between serotype-specific antibodies, transient and long-lasting heterotypic antibodies [105]. Mandatory follow-up studies after the administration of a DENV LAV are equally as important as it is believed that the switch from the protective immunity to the cross-reactive, non-neutralising heterotopic immunity following a natural DENV infection or an incomplete DENV immunisation occurs about 1-2 years following the initial infection or immunisation [106], a problem that is hoped to be solved by the rational design of a DENV LAV.

\section{Competing Interests}

The authors have declared that no competing interest exists.

\section{References}

1. Bhatt S, Gething PW, Brady OJ, Messina JP, Farlow AW, Moyes CL, et al. The global distribution and burden of dengue. Nature. 2013; 496: 504-507.

2. Murray NE, Quam MB, Wilder-Smith A. Epidemiology of dengue: past, present and future prospects. Clin Epidemiol. 2013; 5: 299-309.

3. El Sahili A, Lescar J. Dengue virus non-structural protein 5. Viruses. 2017; 9: 91.

4. Scott LJ. Tetravalent dengue vaccine: A review in the prevention of dengue disease. Drugs. 2016; 76: 1301-1312.

5. Aguiar M, Stollenwerk N, Halstead SB. The impact of the newly licensed dengue vaccine in endemic countries. PLoS Negl Trop Dis. 2016; 10: e0005179.

6. [Internet] WHO: Geneva, Switzerland. Revised SAGE recommendation on use of dengue vaccine. Published 19 April 2018. http://www.who.int/ immunization/diseases/dengue/revised_SAGE_recommendations_dengu e_vaccines_apr2018/en/
7. Wichmann O, Vannice K, Asturias EJ, de Albuquerque Luna EJ, Longini I, Lopez AL, et al. Live-attenuated tetravalent dengue vaccines: The needs and challenges of post-licensure evaluation of vaccine safety and effectiveness. Vaccine. 2017; 35: 5535-5542.

8. Sridhar S, Luedtke A, Langevin E, Zhu M, Bonaparte M, Machabert T, et al. Effect of dengue serostatus on dengue vaccine safety and efficacy. N Engl J Med. 2018; 379: 327-340.

9. Muller DA, Young PR. The flavivirus NS1 protein: molecular and structural biology, immunology, role in pathogenesis and application as a diagnostic biomarker. Antiviral Res. 2013; 98: 192-208.

10. Rivino $\mathrm{L}$. $\mathrm{T}$ cell immunity to dengue virus and implications for vaccine design. Expert Rev Vaccines. 2016; 15: 443-453.

11. Halstead SB. Which dengue vaccine approach is the most promising, and should we be concerned about enhanced disease after vaccination? There is only one true winner. Cold Spring Harb Perspect Biol. 2018; 10: a030700.

12. Ferguson NM. Challenges and opportunities in controlling mosquito-borne infections. Nature. 2018; 559: 490-497.

13. Osorio JE, Huang CY, Kinney RM, Stinchcomb DT. Development of DENVax: a chimeric dengue-2 PDK-53-based tetravalent vaccine for protection against dengue fever. Vaccine. 2011; 29: 7251-7260.

14. Osorio JE, Velez ID, Thomson C, Lopez L, Jimenez A, Haller AA, et al. Safety and immunogenicity of a recombinant live attenuated tetravalent dengue vaccine (DENVax) in flavivirus-naive healthy adults in Colombia: a randomised, placebo-controlled, phase 1 study. Lancet Infect Dis. 2014; 14: 830-838.

15. Saez-Llorens X, Tricou V, Yu D, Rivera L, Tuboi S, Garbes P, et al. Safety and immunogenicity of one versus two doses of Takeda's tetravalent dengue vaccine in children in Asia and Latin America: interim results from a phase 2, randomised, placebo-controlled study. Lancet Infect Dis. 2017; 17: 615-625.

16. [Internet] Takeda: Takeda completes enrolment of more than 20,000 children and adolescents in global phase 3 trial of dengue vaccine candidate. 5 April 2018. https://www.takeda.com/newsroom/newsreleases/2017/takedacompletes-enrollment-of-more-than-20000-children-and-adolescents-in-global -phase-3-trial-of-dengue-vaccine-candidate/

17. Durbin AP, McArthur JH, Marron JA, Blaney JE, Thumar B, Wanionek K, et al. rDEN2/4Delta30(ME), a live attenuated chimeric dengue serotype 2 vaccine is safe and highly immunogenic in healthy dengue-naive adults. Hum Vaccin. 2006; 2: 255-260.

18. Kirkpatrick BD, Durbin AP, Pierce KK, Carmolli MP, Tibery CM, Grier PL, et al. Robust and balanced immune responses to all 4 dengue virus serotypes following administration of a single dose of a live attenuated tetravalent dengue vaccine to healthy, flavivirus-naive adults. J Infect Dis. 2015; 212: 702-710.

19. Kirkpatrick BD, Whitehead SS, Pierce KK, Tibery CM, Grier PL, Hynes NA, et al. The live attenuated dengue vaccine TV003 elicits complete protection against dengue in a human challenge model. Sci Transl Med. 2016; 8: 330ra336.

20. Whitehead SS, Durbin AP, Pierce KK, Elwood D, McElvany BD, Fraser EA, et al. In a randomized trial, the live attenuated tetravalent dengue vaccine TV003 is well-tolerated and highly immunogenic in subjects with flavivirus exposure prior to vaccination. PLoS Negl Trop Dis. 2017; 11: e0005584.

21. Angelo MA, Grifoni A, O'Rourke PH, Sidney J, Paul S, Peters B, et al. Human $\mathrm{CD} 4+\mathrm{T}$ cell responses to an attenuated tetravalent dengue vaccine parallel those induced by natural infection in magnitude, HLA restriction, and antigen specificity. J Virol. 2017; 91: e02147-02116.

22. Butrapet $S$, Kinney RM, Huang $C Y$. Determining genetic stabilities of chimeric dengue vaccine candidates based on dengue 2 PDK-53 virus by sequencing and quantitative TaqMAMA. J Virol Methods. 2006; 131: 1-9.

23. Osorio JE, Wallace D, Stinchcomb DT. A recombinant, chimeric tetravalent dengue vaccine candidate based on a dengue virus serotype 2 backbone. Expert Rev Vaccines. 2016; 15: 497-508.

24. McArthur MA, Edelman R. A promising, single-dose, live attenuated tetravalent dengue vaccine candidate. J Infect Dis. 2015; 212: 681-683.

25. Vannice KS, Roehrig JT, Hombach J. Next generation dengue vaccines: A review of the preclinical development pipeline. Vaccine. 2015; 33: 7091-7099.

26. Hadinegoro SR, Arredondo-Garcia JL, Capeding MR, Deseda C, Chotpitayasunondh T, Dietze R, et al. Efficacy and long-term safety of a dengue vaccine in regions of endemic disease. N Engl J Med. 2015; 373: 1195-1206.

27. Kanesa-thasan N, Sun W, Kim-Ahn G, Van Albert S, Putnak JR, King A, et al. Safety and immunogenicity of attenuated dengue virus vaccines (Aventis Pasteur) in human volunteers. Vaccine. 2001; 19: 3179-3188.

28. Balas C, Kennel A, Deauvieau F, Sodoyer R, Arnaud-Barbe N, Lang J, et al. Different innate signatures induced in human monocyte-derived dendritic cells by wild-type dengue 3 virus, attenuated but reactogenic dengue 3 vaccine virus, or attenuated nonreactogenic dengue 1-4 vaccine virus strains. J Infect Dis. 2011; 203: 103-108.

29. Sanchez V, Gimenez S, Tomlinson B, Chan PK, Thomas GN, Forrat R, et al. Innate and adaptive cellular immunity in flavivirus-naive human recipients of a live-attenuated dengue serotype 3 vaccine produced in Vero cells (VDV3). Vaccine. 2006; 24: 4914-4926.

30. Edelman R, Wasserman SS, Bodison SA, Putnak RJ, Eckels KH, Tang D, et al. Phase I trial of 16 formulations of a tetravalent live-attenuated dengue vaccine. Am J Trop Med Hyg. 2003; 69: 48-60. 
31. Guy B, Barban V, Mantel N, Aguirre M, Gulia S, Pontvianne J, et al. Evaluation of interferences between dengue vaccine serotypes in a monkey model. Am J Trop Med Hyg. 2009; 80: 302-311.

32. Blok J. Genetic relationships of the dengue virus serotypes. J Gen Virol. 1985; 66 (Pt 6): 1323-1325.

33. Chawla T, Chan KR, Zhang SL, Tan HC, Lim AP, Hanson BJ, et al. Dengue virus neutralization in cells expressing Fc gamma receptors. PLoS One. 2013; 8: e65231.

34. Kliks SC, Nimmanitya S, Nisalak A, Burke DS. Evidence that maternal dengue antibodies are important in the development of dengue hemorrhagic fever in infants. Am J Trop Med Hyg. 1988; 38: 411-419.

35. Diamond Michael S, Pierson Theodore C. Molecular insight into dengue virus pathogenesis and its implications for disease control. Cell. 2015; 162: 488-492.

36. Kellman EM, Offerdahl DK, Melik W, Bloom ME. Viral determinants of virulence in tick-borne flaviviruses. Viruses. 2018; 10: 329.

37. Guzman MG, Harris E. Dengue. Lancet. 2015; 385: 453-465.

38. Markoff L. 5'- and 3'-noncoding regions in flavivirus RNA. Adv Virus Res. 2003; 59: 177-228.

39. Brinton MA, Dispoto JH. Sequence and secondary structure analysis of the 5'-terminal region of flavivirus genome RNA. Virology. 1988; 162: 290-299.

40. Cahour A, Pletnev A, Vazielle-Falcoz M, Rosen L, Lai CJ. Growth-restricted dengue virus mutants containing deletions in the $5^{\prime}$ noncoding region of the RNA genome. Virology. 1995; 207: 68-76.

41. Filomatori CV, Lodeiro MF, Alvarez DE, Samsa MM, Pietrasanta L, Gamarnik AV. A 5' RNA element promotes dengue virus RNA synthesis on a circular genome. Genes Dev. 2006; 20: 2238-2249.

42. Alvarez DE, De Lella Ezcurra AL, Fucito S, Gamarnik AV. Role of RNA structures present at the 3'UTR of dengue virus on translation, RNA synthesis, and viral replication. Virology. 2005; 339: 200-212.

43. Sirigulpanit W, Kinney RM, Leardkamolkarn V. Substitution or deletion mutations between nt 54 and 70 in the 5 ' non-coding region of dengue type 2 virus produce variable effects on virus viability. J Gen Virol. 2007; 88: $1748-1752$.

44. Leitmeyer KC, Vaughn DW, Watts DM, Salas R, Villalobos I, de C, et al. Dengue virus structural differences that correlate with pathogenesis. J Virol. 1999; 73: 4738-4747.

45. Zhu W, Qin C, Chen S, Jiang T, Yu M, Yu X, et al. Attenuated dengue 2 viruses with deletions in capsid protein derived from an infectious full-length cDNA clone. Virus Res. 2007; 126: 226-232.

46. Clyde K, Kyle JL, Harris E. Recent advances in deciphering viral and host determinants of dengue virus replication and pathogenesis. J Virol. 2006; 80: 11418-11431.

47. Clyde K, Barrera J, Harris E. The capsid-coding region hairpin element (cHP) is a critical determinant of dengue virus and West Nile virus RNA synthesis. Virology. 2008; 379: 314-323.

48. Pryor MJ, Azzola L, Wright PJ, Davidson AD. Histidine 39 in the dengue virus type $2 \mathrm{M}$ protein has an important role in virus assembly. J Gen Virol. 2004; 85: $3627-3636$.

49. Guirakhoo F, Arroyo J, Pugachev KV, Miller C, Zhang ZX, Weltzin R, et al. Construction, safety, and immunogenicity in nonhuman primates of a chimeric yellow fever-dengue virus tetravalent vaccine. J Virol. 2001; 75: 7290-7304.

50. Wang S, He R, Anderson R. prM- and cell-binding domains of the dengue virus E protein. J Virol. 1999; 73: 2547-2551.

51. Hsieh SC, Zou G, Tsai WY, Oing M, Chang GJ, Shi PY, et al. The C-terminal helical domain of dengue virus precursor membrane protein is involved in virus assembly and entry. Virology. 2011; 410: 170-180.

52. Hsieh SC, Wu YC, Zou G, Nerurkar VR, Shi PY, Wang WK. Highly conserved residues in the helical domain of dengue virus type 1 precursor membrane protein are involved in assembly, precursor membrane (prM) protein cleavage and entry. J Biol Chem. 2014; 289: 33149-33160.

53. Zybert IA, van der Ende-Metselaar H, Wilschut J, Smit JM. Functional importance of dengue virus maturation: infectious properties of immature virions. J Gen Virol. 2008; 89: 3047-3051.

54. Kroeger MA, McMinn PC. Murray Valley encephalitis virus recombinant subviral particles protect mice from lethal challenge with virulent wild-type virus. Arch Virol. 2002; 147: 1155-1172.

55. Konishi E, Fujii A. Dengue type 2 virus subviral extracellular particles produced by a stably transfected mammalian cell line and their evaluation for a subunit vaccine. Vaccine. 2002; 20: 1058-1067.

56. Butrapet S, Childers T, Moss KJ, Erb SM, Luy BE, Calvert AE, et al. Amino acid changes within the $\mathrm{E}$ protein hinge region that affect dengue virus type 2 infectivity and fusion. Virology. 2011; 413: 118-127.

57. Ni H, Ryman KD, Wang H, Saeed MF, Hull R, Wood D, et al. Interaction of yellow fever virus French neurotropic vaccine strain with monkey brain: characterization of monkey brain membrane receptor escape variants. J Virol. 2000; 74: 2903-2906.

58. Wang E, Ryman KD, Jennings AD, Wood DJ, Taffs F, Minor PD, et al. Comparison of the genomes of the wild-type French viscerotropic strain of yellow fever virus with its vaccine derivative French neurotropic vaccine. J Gen Virol. 1995; 76 2749-2755.

59. Huang CY, Butrapet S, Moss KJ, Childers T, Erb SM, Calvert AE, et al. The dengue virus type 2 envelope protein fusion peptide is essential for membrane fusion. Virology. 2010; 396: 305-315.
60. Smit JM, Moesker B, Rodenhuis-Zybert I, Wilschut J. Flavivirus cell entry and membrane fusion. Viruses. 2011; 3: 160-171.

61. Chotiwan N, Roehrig JT, Schlesinger JJ, Blair CD, Huang CY. Molecular determinants of dengue virus 2 envelope protein important for virus entry in FcyRIIA-mediated antibody-dependent enhancement of infection. Virology. 2014; 456: 238-246.

62. Zhang Y, Zhang W, Ogata S, Clements D, Strauss JH, Baker TS, et al. Conformational changes of the flavivirus E glycoprotein. Structure. 2004; 12: $1607-1618$

63. Modis Y, Ogata S, Clements D, Harrison SC. Structure of the dengue virus envelope protein after membrane fusion. Nature. 2004; 427: 313-319.

64. Goo L, VanBlargan LA, Dowd KA, Diamond MS, Pierson TC. A single mutation in the envelope protein modulates flavivirus antigenicity, stability, and pathogenesis. PLoS Pathog. 2017; 13: e1006178.

65. Oliphant T, Nybakken GE, Engle M, Xu Q, Nelson CA, Sukupolvi-Petty S, et al. Antibody recognition and neutralization determinants on domains I and II of West Nile Virus envelope protein. J Virol. 2006; 80: 12149-12159.

66. Winkler G, Randolph VB, Cleaves GR, Ryan TE, Stollar V. Evidence that the mature form of the Flavivirus nonstructural protein NS1 is a dimer. Virology. 1988; 162: 187-196.

67. Xie X, Gayen S, Kang C, Yuan Z, Shi PY. Membrane topology and function of dengue virus NS2A protein. J Virol. 2013; 87: 4609-4622.

68. Leung JY, Pijlman GP, Kondratieva N, Hyde J, Mackenzie JM, Khromykh AA. Role of nonstructural protein NS2A in flavivirus assembly. J Virol. 2008; 82: 4731-4741.

69. Mackenzie JM, Jones MK, Young PR. Immunolocalization of the dengue virus nonstructural glycoprotein NS1 suggests a role in viral RNA replication. Virology. 1996; 220: 232-240.

70. Scaturro P, Cortese M, Chatel-Chaix L, Fischl W, Bartenschlager R. Dengue virus non-structural protein 1 modulates infectious particle production via interaction with the structural proteins. PLoS Pathog. 2015; 11: e1005277.

71. Akey DL, Brown WC, Dutta S, Konwerski J, Jose J, Jurkiw TJ, et al. Flavivirus NS1 crystal structures reveal a surface for membrane association and regions of interaction with the immune system. Science. 2014; 343: 881-885.

72. Xie X, Zou J, Puttikhunt C, Yuan Z, Shi PY. Two distinct sets of NS2A molecules are responsible for dengue virus RNA synthesis and virion assembly. J Virol. 2015; 89: 1298-1313.

73. Wu RH, Tsai MH, Tsai KN, Tian JN, Wu JS, Wu SY, et al. Mutagenesis of dengue virus protein NS2A revealed a novel domain responsible for virus-induced cytopathic effect and interactions between NS2A and NS2B transmembrane segments. J Virol. 2017; 91: e01836-01816.

74. Falgout B, Miller RH, Lai CJ. Deletion analysis of dengue virus type 4 nonstructural protein NS2B: identification of a domain required for NS2B-NS3 protease activity. J Virol. 1993; 67: 2034-2042.

75. Li J, Lim SP, Beer D, Patel V, Wen D, Tumanut C, et al. Functional profiling of recombinant NS3 proteases from all four serotypes of dengue virus using tetrapeptide and octapeptide substrate libraries. J Biol Chem. 2005; 280: 28766-28774.

76. Li Y, Kim YM, Zou J, Wang QY, Gayen S, Wong YL, et al. Secondary structure and membrane topology of dengue virus NS4B N-terminal 125 amino acids. Biochim Biophys Acta. 2015; 1848: 3150-3157.

77. Luo D, Vasudevan SG, Lescar J. The flavivirus NS2B-NS3 protease-helicase as a target for antiviral drug development. Antiviral Res. 2015; 118: 148-158.

78. Luo D, Xu T, Watson RP, Scherer-Becker D, Sampath A, Jahnke W, et al. Insights into RNA unwinding and ATP hydrolysis by the flavivirus NS3 protein. Embo J. 2008; 27: 3209-3219.

79. Blaney JE, Jr., Johnson DH, Manipon GG, Firestone CY, Hanson CT, Murphy $\mathrm{BR}$, et al. Genetic basis of attenuation of dengue virus type 4 small plaque mutants with restricted replication in suckling mice and in SCID mice transplanted with human liver cells. Virology. 2002; 300: 125-139.

80. Miller S, Kastner S, Krijnse-Locker J, Buhler S, Bartenschlager R. The non-structural protein $4 \mathrm{~A}$ of dengue virus is an integral membrane protein inducing membrane alterations in a $2 \mathrm{~K}$-regulated manner. J Biol Chem. 2007; 282: $8873-8882$

81. Stern O, Hung YF, Valdau O, Yaffe $Y$, Harris E, Hoffmann $S$, et al. An $\mathrm{N}$-terminal amphipathic helix in dengue virus nonstructural protein $4 \mathrm{~A}$ mediates oligomerization and is essential for replication. J Virol. 2013; 87: 4080-4085.

82. Lee CM, Xie X, Zou J, Li SH, Lee MY, Dong H, et al. Determinants of dengue virus NS4A protein oligomerization. J Virol. 2015; 89: 6171-6183.

83. Zou J, Lee le T, Wang QY, Xie X, Lu S, Yau YH, et al. Mapping the interactions between the NS4B and NS3 proteins of dengue virus. J Virol. 2015; 89: 3471-3483

84. Miller S, Sparacio S, Bartenschlager R. Subcellular localization and membrane topology of the dengue virus type 2 non-structural protein 4B. J Biol Chem. 2006; $281: 8854-8863$.

85. Naik NG, Wu HN. Mutation of putative N-glycosylation sites on dengue virus NS4B decreases RNA replication. J Virol. 2015; 89: 6746-6760.

86. Hanley KA, Manlucu LR, Gilmore LE, Blaney JE, Jr., Hanson CT, Murphy BR, et al. A trade-off in replication in mosquito versus mammalian systems conferred by a point mutation in the NS4B protein of dengue virus type 4 . Virology. 2003; 312: 222-232.

87. Liu L, Dong H, Chen H, Zhang J, Ling H, Li Z, et al. Flavivirus RNA cap methyltransferase: structure, function, and inhibition. Front Biol. 2010; 5: 286-303. 
88. Ferron F, Decroly E, Selisko B, Canard B. The viral RNA capping machinery as a target for antiviral drugs. Antiviral Res. 2012; 96: 21-31.

89. Mazzon M, Jones M, Davidson A, Chain B, Jacobs M. Dengue virus NS5 inhibits interferon-alpha signaling by blocking signal transducer and activator of transcription 2 phosphorylation. J Infect Dis. 2009; 200: 1261-1270.

90. Yon C, Teramoto T, Mueller N, Phelan J, Ganesh VK, Murthy KH, et al. Modulation of the nucleoside triphosphatase/RNA helicase and 5'-RNA triphosphatase activities of dengue virus type 2 nonstructural protein 3 (NS3) by interaction with NS5, the RNA-dependent RNA polymerase. J Biol Chem. 2005; 280: 27412-27419.

91. Iglesias NG, Filomatori CV, Gamarnik AV. The F1 motif of dengue virus polymerase NS5 is involved in promoter-dependent RNA synthesis. J Virol. 2011; 85: 5745-5756.

92. Filomatori CV, Iglesias NG, Villordo SM, Alvarez DE, Gamarnik AV. RNA sequences and structures required for the recruitment and activity of the dengue virus polymerase. J Biol Chem. 2011; 286: 6929-6939.

93. McMinn PC. The molecular basis of virulence of the encephalitogenic flaviviruses. J Gen Virol. 1997; 78 2711-2722.

94. Ng WC, Soto-Acosta R, Bradrick SS, Garcia-Blanco MA, Ooi EE. The 5' and $3^{\prime}$ untranslated regions of the flaviviral genome. Viruses. 2017; 9: 137.

95. Manzano M, Reichert ED, Polo S, Falgout B, Kasprzak W, Shapiro BA, et al. Identification of cis-acting elements in the 3 -untranslated region of the dengue virus type 2 RNA that modulate translation and replication. J Biol Chem. 2011; 286: 22521-22534.

96. Proutski V, Gritsun TS, Gould EA, Holmes EC. Biological consequences of deletions within the 3'-untranslated region of flaviviruses may be due to rearrangements of RNA secondary structure. Virus Res. 1999; 64: 107-123.

97. Kieft JS, Rabe JL, Chapman EG. New hypotheses derived from the structure of a flaviviral Xrn1-resistant RNA: Conservation, folding, and host adaptation. RNA Biol. 2015; 12: 1169-1177.

98. Clarke BD, Roby JA, Slonchak A, Khromykh AA. Functional non-coding RNAs derived from the flavivirus 3' untranslated region. Virus Res. 2015; 206: 53-61.

99. Filomatori CV, Carballeda JM, Villordo SM, Aguirre S, Pallarés HM, Maestre $\mathrm{AM}$, et al. Dengue virus genomic variation associated with mosquito adaptation defines the pattern of viral non-coding RNAs and fitness in human cells. PLoS Pathogens. 2017; 13: e1006265-e1006265.

100. Carmo AM, Suzuki RB, Cabral AD, Costa RT, Massari GP, Riquena MM, et al. Co-circulating serotypes in a dengue fever outbreak: Differential hematological profiles and phylogenetic relationships among viruses. J Clin Virol. 2017; 90: 7-13.

101. Durbin AP, Kirkpatrick BD, Pierce KK, Schmidt AC, Whitehead SS. Development and clinical evaluation of multiple investigational monovalent DENV vaccines to identify components for inclusion in a live attenuated tetravalent DENV vaccine. Vaccine. 2011; 29: 7242-7250.

102. Weiskopf D, Angelo MA, de Azeredo EL, Sidney J, Greenbaum JA, Fernando AN, et al. Comprehensive analysis of dengue virus-specific responses supports an HLA-linked protective role for CD8+ T cells. Proc Natl Acad Sci U S A. 2013; 110: E2046-2053.

103. Weiskopf D, Angelo MA, Bangs DJ, Sidney J, Paul S, Peters B, et al. The human CD8 + T cell responses induced by a live attenuated tetravalent dengue vaccine are directed against highly conserved epitopes. J Virol. 2015; 89: 120-128.

104. Whitehead SS, Blaney JE, Durbin AP, Murphy BR. Prospects for a dengue virus vaccine. Nat Rev Microbiol. 2007; 5: 518-528.

105. Vannice KS, Wilder-Smith A, Barrett ADT, Carrijo K, Cavaleri M, de Silva A, et al. Clinical development and regulatory points for consideration for second-generation live attenuated dengue vaccines. Vaccine. 2018; 36: 3411-3417.

106. Montoya M, Gresh L, Mercado JC, Williams KL, Vargas MJ, Gutierrez G, et al. Symptomatic versus inapparent outcome in repeat dengue virus infections is influenced by the time interval between infections and study year. PLoS Negl Trop Dis. 2013; 7: e2357. 